\title{
Health Risk Behaviors and Prostate-Specific Antigen Awareness Among California Men
}

\author{
Firas S. Ahmed, MD, MPH ${ }^{1,2}$, Luisa N. Borrell, DDS, PhD ${ }^{2,3}$, and Benjamin A. Spencer, MD, \\ $\mathrm{MPH}^{2,4}$ \\ 1 Department of Medicine, Division of General Medicine, College of Physicians and Surgeons, Columbia \\ University \\ 2Department of Epidemiology, Mailman School of Public Health, Columbia University \\ 3Department of Health Sciences, Lehman College, City University of New York \\ 4Department of Urology, College of Physicians and Surgeons, Columbia University
}

\begin{abstract}
Purpose-Differences in prostate-specific antigen (PSA) awareness may contribute to differences in frequency of PSA testing. We investigated the association of health risk behaviors, including smoking, physical inactivity, obesity, and excessive alcohol consumption with awareness of the PSA test among California men at risk for prostate cancer.
\end{abstract}

Materials and Methods-Using California Health Interview Survey (CHIS) 2003 data, a population-based random-digit-dial telephone survey, records of 7,297 men aged 50 years and older without a prior history of prostate cancer were identified. The outcome was self-reported awareness of the PSA test. The main independent variables were smoking status, level of physical activity, body mass index (BMI), and alcohol consumption. Prevalence, odds ratios (OR) and 95\% confidence intervals (CI) for PSA awareness were calculated using SUDAAN to account for the complex sampling design.

Results-The overall prevalence of PSA awareness was 73.0\%. After controlling for potential confounders, the odds of being aware of the PSA test was lower among current smokers ( $\mathrm{OR}=0.53$; $95 \% \mathrm{CI}=0.41-0.68$ vs. never smoked), physically inactive $(\mathrm{OR}=0.77 ; 95 \% \mathrm{CI}=0.63-0.93$ vs. physically active) and obese men ( $\mathrm{OR}=0.77 ; 95 \% \mathrm{CI}=0.62-0.95$ vs. normal weight).

Conclusions-Health risk behaviors are associated with lower PSA awareness. Our findings suggest opportunities for focused health education interventions and quality improvement programs tailored to men who engage in unhealthy behaviors to improve their PSA test awareness.

\section{Keywords}

Prostate-Specific Antigen; Awareness; Smoking; Obesity; Exercise

\footnotetext{
(c) 2009 American Urological Association. Published by Elsevier Inc. All rights reserved.

Corresponding Author: Firas S. Ahmed MD, MPH Columbia University Medical Center 622 West 168th St, PH 9 East, Room 109 New York, NY 10032 Office: 212-305-3073 Fax: 212-305-9349.

Publisher's Disclaimer: This is a PDF file of an unedited manuscript that has been accepted for publication. As a service to our customers we are providing this early version of the manuscript. The manuscript will undergo copyediting, typesetting, and review of the resulting proof before it is published in its final citable form. Please note that during the production process errors may be discovered which could affect the content, and all legal disclaimers that apply to the journal pertain.
} 


\section{INTRODUCTION}

Health risk behaviors such as smoking and obesity have been found to be associated with higher prostate cancer morbidity and mortality. Evidence suggests that prostate cancer patients who smoke present with more advanced stages of prostate cancer, greater risk of metastases, and worse prognosis compared to patients who do not smoke. ${ }^{1}$ A growing body of research suggests that obesity is associated with more advanced stages and higher grades of prostate cancer. ${ }^{2}$,

${ }^{3}$ Regular physical activity may lower the risk of prostate cancer, ${ }^{4}$ slow its progression, and ultimately lower prostate cancer mortality. 5

A recent study demonstrated that persons who seek out cancer information are more likely to acquire knowledge, display healthy behaviors, and undergo cancer screening. ${ }^{6}$ Awareness of PSA testing is regarded as an "important cognitive precursor" of prostate cancer screening and was found to contribute to differences in prostate cancer screening rates. ${ }^{7}$ Nevertheless, one in four men above the age of 50 remains unaware of the PSA test. 8 Previous studies have explored predictors of PSA test awareness, 9,10 but none has focused on health risk behaviors. With greater appreciation of the impact of health behaviors on cancer prevention, ${ }^{11}$ we sought to investigate the association between selected health risk behaviors and awareness of the PSA test. We hypothesized that men reporting health risk behaviors have less awareness of the PSA test.

\section{MATERIALS AND METHODS}

The 2003 California Health Interview Survey (CHIS), a population-based random digit dialing telephone survey conducted between August, 2003 and February, 2004, is the largest state health-related survey conducted in the U.S. It used a two-stage sampling approach to randomly select phone numbers from 41 geographic sampling strata representative of California's population. Phone numbers were screened to confirm that they were related to residential households. Interviews were conducted in English, Spanish, Chinese, Vietnamese, and Korean. The adult interview response rate was $60 \%,{ }^{12}$ a rate comparable to the California Behavioral Risk Factor Surveillance System.

The target population for the survey was adults aged 18 years and older living in California in $2003(n=42,044)$. For this analysis the sample was limited to men aged 50 years and older to reflect the joint American Cancer Society/American Urological Association recommendation to offer prostate cancer screening after informed consent. ${ }^{13}$ Men with a prior history of prostate cancer were excluded from the study to avoid selection bias. These exclusion criteria yielded a final sample size of 7,297 men. Due to missing data, an additional 178 records were excluded from the final multivariable logistic regression.

\section{Exposure and Outcome Variables}

PSA awareness, the study outcome, was determined from the question "Have you ever heard of the PSA test?" The independent variables included in the analysis were: smoking status, walking (a measure of physical activity), BMI (a measure of obesity), and binge alcohol drinking. Smoking status was specified as current, former or never smoked. Because assessing physical activity through self-reported measures may lead to misclassification, we used a conservative definition of physical activity that allows identification of men with sedentary life-styles. Self-reported physical activity was defined as walking for transport or fun/exercise for at least 10 minutes within the past week and it was coded as a binary variable (yes/no). BMI was categorized according to the International Classification of Adult Body Mass Index as underweight $\left(<18.5 \mathrm{~kg} / \mathrm{m}^{2}\right)$, normal weight $\left(18.5-24.9 \mathrm{~kg} / \mathrm{m}^{2}\right)$, overweight $(25-29.9 \mathrm{~kg} /$ $\left.\mathrm{m}^{2}\right)$, and obese $\left(>30.0 \mathrm{~kg} / \mathrm{m}^{2}\right)$. Since drinking in moderation has a cardiac protective effect, 14 we were interested in excessive alcohol consumption as a health risk behavior. Thus, we 
used binge drinking, a binary variable that required having at least 5 drinks per occasion within the past 30 days, to identify men with unhealthy alcohol intake.

\section{Potential Confounders}

Based on prior studies demonstrating relationships with PSA testing $7-9,15$ which is likely highly correlated with PSA awareness, we included several potential confounding variables, including demographics, socioeconomic status, access to healthcare, health status and colon cancer screening. Age was classified into five-year intervals and greater than 70 years old. Race and ethnicity were categorized as: White, African American, Latino, Asian, American Indian/Alaska Native, Pacific Islander and Other Races. Acculturation was represented by English proficiency (speaks only English, speaks English well/very well, speaks English not well/not at all) and the percentage of an individual's lifetime spent in the U.S. (categorized in quintiles: $\leq 20 \%, 21-40 \%, 41-60 \%, 61-80 \%$, and $\geq 80 \%$ of life time). Socioeconomic indicators were annual household income as a percentage of the Federal Poverty Level (\%FPL), educational attainment, and marital status. The \%FPL (calculated from the total annual household income, family size, and the 2002 federal poverty guidelines) was categorized into four categories: below 100\%, 100-199\%, 200-299\%, and $\geq 300 \%$ FPL. Education was specified as four levels: less than high school, high school graduate, some college, and college or higher education. Marital status was categorized into three levels: married, never married, and others.

Access to healthcare measures included health insurance status during the past year (uninsured for the whole year, uninsured for part of the year, insured), number of physician visits within the past year (zero, one to two times, three to four times, five times and above), and having a usual source of care other than an emergency room (ER) (yes/no). Self-reported health status was categorized into five levels (excellent, very good, good, fair, and poor). Finally, colorectal cancer screening behavior (defined as having a colonoscopy, sigmoidoscopy or fecal occult blood test) was categorized as screened within past 5 years, not screened within past five years, and never screened.

\section{Statistical Analysis}

Descriptive statistics were used to examine the characteristics of the sample and the prevalence of PSA awareness. To test for significant differences in PSA awareness across levels of each characteristic (covariate), we used the chi-square statistic. Logistic regression models were implemented to estimate the association of each risk behavior (smoking, physical inactivity, obesity, and binge drinking) with the participants' awareness of PSA before and after adjusting for selected covariates. To test whether the strength of the association between each health risk behavior and awareness of PSA test differs by age, race/ethnicity and income, interaction terms were tested. A sensitivity analysis was conducted including BMI as a continuous variable and a quadratic term to evaluate for possible curvilinearity or a threshold effect of BMI on awareness of the PSA test.

Since health risk behaviors tend to coexist, ${ }^{16}$ we tested whether the presence of more than one risk behavior would incrementally affect awareness of the PSA test. We created a variable representing the number of health risk behaviors for each individual, ranging from zero (for men with no health risk behavior) to three (for men having three or more risk behaviors). We measured the prevalence of PSA awareness across levels of this variable and tested the association between this variable and PSA awareness while adjusting for other covariates.

Data management was conducted using SAS (version 9.1; SAS Institute Inc., Cary, NC) while all analyses were performed using SUDAAN (Research Triangle Institute, Research Triangle Park, NC), which takes into account the complex sampling design and yields unbiased standard 
error estimates. Estimates for percentages, prevalence and odds ratios (ORs) with their 95\% confidence intervals (CI) are weighted.

\section{RESULTS}

Characteristics of the study population are shown in Table 1. Nearly one-half of the subjects were between the ages of 50 and 60. The majority were white, married, spoke only English, and up-to-date for colorectal cancer screening; lived most of their lives in the US; reported their health status as good or better; had income of at least 300\% of FPL, had at least some college education, health insurance, and a usual source of medical care.

Overall, the prevalence of PSA awareness for the study population is $73 \%$ while the prevalence of PSA testing is $39 \%$ (data not shown). The prevalence of PSA awareness increases with age up to 70 years old and then declines. PSA awareness was highest among whites, Pacific Islanders and American Indians and lowest among Asians; Latinos and African Americans exhibited an intermediate PSA awareness. The prevalence of PSA awareness was positively associated with education, income, health status, and number of physician visits in the past year. Those with health insurance, a usual source of healthcare other than the ER, and recent colorectal cancer screening had higher prevalence of PSA awareness than those lacking such resources.

Table 2 shows the ORs (crude and adjusted) with their 95\% CI for the association between health risk behaviors and PSA awareness. After adjusting for all covariates, smoking, physical inactivity and obesity were significantly associated with lower odds of PSA awareness. Specifically, current smokers $(\mathbf{O R}=\mathbf{0 . 5 3}$; 95\% CI: 0.41-0.68), physically inactive men (OR=0.77; 95\% CI: 0.63-0.93), and obese men $(\mathrm{OR}=0.77$; 95\%CI: $0.62-0.95)$ had a lower odds of being aware of the PSA test than those who never smoked, were physically active and had normal weight, respectively.

There were neither interactions observed between age, race/ethnicity or level of income and any of the risk behaviors, nor significant confounding by any of the covariates, except for partial negative confounding of the association between BMI and PSA awareness by race.

Finally, to measure the effect of clustering of risk behaviors in an individual, we examined the prevalence of PSA awareness among men with one to four risk behaviors as compared to men without any risk behaviors. The prevalence of PSA awareness decreased from $78 \%$ among men with no risk behaviors to $71 \%, 68 \%$ and $55 \%$ among men with one, two, and three or more risk behaviors, respectively. After adjusting for the covariates, the odds of PSA awareness among men with one (OR=0.76; 95\%CI: $0.61-0.93)$, two (OR=0.69; 95\%CI: $0.54-0.89)$, and three or more risk behaviors $(\mathrm{OR}=0.38 ; 95 \% \mathrm{CI}$ : $0.26-0.56)$ was significantly lower than men with no risk behaviors in a dose-dependent manner (Figure 1).

\section{DISCUSSION}

Our primary findings suggest that smoking, physical inactivity, and obesity are inversely associated with awareness of the PSA test. No such association with excessive alcohol drinking was identified. Furthermore, clustering of more than one risk behavior in the same individual decreased the odds of PSA awareness in a dose-dependent manner. These results concur with our initial hypothesis that men who adopt unhealthy lifestyles may be less concerned with their health and less aware of preventive measures like the PSA test.

Intuitively, a person who is unaware of the presence of a screening test would be less likely to undergo screening. McFall demonstrated that a lack of PSA awareness contributes to lower rates of PSA test use among racial minorities. ${ }^{7}$ We have extended the analysis of PSA 
awareness to certain health risk behaviors, each of which has been previously shown to be associated with PSA testing. 9,15

An Australian study found that men who smoke were less likely to undergo PSA screening than non-smokers. ${ }^{17}$ This difference in PSA screening rates may be explained by the differences in awareness of the PSA test. Although we did not use PSA testing as an outcome measure, our results agree with the Australian study's finding in showing that current smokers are the least aware of PSA, followed by former smokers and men who never smoked. It is possible that this finding may be due to a general lack of concern about health maintenance or less interactions with healthcare providers among smokers. Lower awareness of the PSA test and ultimately less PSA screening among smokers warrants a focused approach by healthcare providers to offer information on the PSA test and the opportunity for prostate cancer screening.

Close showed that regular physical exercise in men was positively associated with the probability of being screened for prostate cancer. ${ }^{18}$ Our results parallel this finding in showing that the odds of PSA test awareness among physically active men was higher than those with sedentary lifestyles, though the magnitude of the association diminished after adjusting for covariates. It is plausible that men with healthy behaviors (regular exercise) may be more concerned about their health and more eager to learn about screening tests. Since there is evidence that physical inactivity is associated with increased risk of prostate cancer, ${ }^{4}$ physically inactive men may constitute a particularly high-risk group in that they are both less aware of screening for and more prone to developing prostate cancer.

A recent study by Scales demonstrated that overweight and obese men are preferentially tested for PSA. ${ }^{15}$ Although we found a similar pattern in our unadjusted analyses which is contrary to our hypothesized lower awareness among obese individuals, this pattern was reversed after controlling for the potential confounders. It is possible that the discrepancy may be due to difference in the outcomes: Scales focused on self-reported PSA testing while we focused on awareness of the PSA test. Reliance on participants' memories to recall PSA testing may result in measurement error which could be alleviated by reviewing medical records. Several studies have demonstrated that self-reported PSA testing is not a sensitive measure of actual PSA testing ${ }^{19}$ and that as many as two-thirds of men referred to a urologist because of high PSA levels were unaware that their PSA level had been measured. ${ }^{20}$ To verify our BMI results, a sensitivity analysis (with BMI as a continuous variable in the prediction model) was conducted and consistently demonstrated an inverse association between BMI and PSA test awareness.

Several studies have demonstrated that health risk behaviors cluster in adults. ${ }^{16}$ Our analysis showed that clustering of more than one health risk behavior is associated with an incremental decline in the odds of PSA awareness. This finding agrees with our initial hypothesis that accumulating risk behaviors would be inversely associated with awareness of cancer preventive approach. Given the associations between smoking, physical activity and obesity with both prostate cancer and cardiovascular disease, men with multiple risk behaviors would seem to be ideal targets for interventions to improve their awareness of the PSA test.

There are several limitations to our study. Due to its cross-sectional design, our study cannot determine temporality between the risk factors and outcome and therefore invoke causality between them. In addition, the study is subject to measurement errors or recall bias since it is based on a self-reported phone survey. Risk behaviors like smoking, obesity, and excessive alcohol drinking are particularly prone to under-reporting and therefore our results may underestimate the true effects. Finally, using an odds ratio as a measure of association for a common outcome may overestimate the effect size. On the other hand, our study does have several methodological advantages including its adjustment for possible confounders, robust 
statistical methods, large sample size, and weighting of the data to extrapolate to the state of California.

\section{CONCLUSION}

This study demonstrates that health risk behaviors are associated with lower awareness of the PSA test which may lead to a lower likelihood of undergoing actual prostate cancer screening. Coupling greater cardiovascular co-morbidities with higher risk disease places these men in a particularly high-risk group. These men would likely benefit from focused health education and counseling regarding PSA testing. Healthcare providers should be aware of such knowledge deficits and the concomitant effects they may have on cancer screening.

\section{Acknowledgements}

The research was supported by

a. Fulbright Scholarship/Institute of International Education (FSA).

b. The National Institute of Health, National Institute of Dental and Craniofacial Research (LNB), Robert Wood Johnson Foundation (LNB).

c. The Department of Defense Congressionally-Directed Medical Research Program, Prostate Cancer Research Program (PC 040167) (BAS).

\section{Glossary}

PSA, Prostate-Specific Antigen; BMI, Body Mass Index; CHIS, California Health and Interview Survey; ACS, American Cancer Society; ER, Emergency Room; OR, Odds Ratio; CI, Confidence Interval; UCLA, University of California, Los Angeles; FPL, Federal Poverty Level.

\section{REFERENCES}

1. Daniell HW. A worse prognosis for smokers with prostate cancer. J Urol 1995;154(1):153-7. [PubMed: 7776411]

2. Freedland SJ, Terris MK, Platz EA, Presti JC Jr. Body mass index as a predictor of prostate cancer: development versus detection on biopsy. Urology 2005;66(1):108-13. [PubMed: 15992911]

3. Gong Z, Agalliu I, Lin DW, Stanford JL, Kristal AR. Obesity is associated with increased risks of prostate cancer metastasis and death after initial cancer diagnosis in middle-aged men. Cancer 2007;109(6):1192-202. [PubMed: 17311344]

4. Krishnadasan A, Kennedy N, Zhao Y, Morgenstern H, Ritz B. Nested case-control study of occupational physical activity and prostate cancer among workers using a job exposure matrix. Cancer Causes Control 2008;19:107-14. [PubMed: 18064535]

5. Giovannucci EL, Liu Y, Leitzmann MF, Stampfer MJ, WC. W. A prospective study of physical activity and incident and fatal prostate cancer. Archives of Internal Medicine 2005;165(9):1005-10. [PubMed: 15883238]

6. Shim M, Kelly B, Hornik R. Cancer information scanning and seeking behavior is associated with knowledge, lifestyle choices, and screening. J Health Commun 2006;11(Suppl 1):157-72. [PubMed: 16641081]

7. McFall SL. Use and Awareness of Prostate Specific Antigen Tests and Race/Ethnicity. the Journal of Urology 2007;177:1475-80. [PubMed: 17382758]

8. Spencer BA, Babey SH, Etzioni DA, Ponce NA, Brown ER, Yu H, et al. A population-based survey of prostate-specific antigen testing among California men at higher risk for prostate carcinoma. Cancer 2006;106(4):765-74. [PubMed: 16419068]

9. Norris CG. Lifestyle and clinical health behaviors and PSA tests. American Journal of Health Education 2006;37(5):289-95.MS 
10. Ross LE, Uhler RJ, Williams KN. Awareness and use of the prostate-specific antigen test among African-American men. J Natl Med Assoc 2005;97(7):963-71. [PubMed: 16080666]

11. Gotay CC. Behavior and cancer prevention. J Clin Oncol 2005;23(2):301-10. [PubMed: 15637393]

12. Public Use File (PUF) Dictionary. (Accessed at http://www.chis.ucla.edu/main/PUF/puf03_adult_datadic.pdf.)

13. Detailed Guide: Prostate Cancer Can Prostate Cancer Be Found Early?. Jul. 2006 (Accessed at http://www.cancer.org/docroot/CRI/content/CRI_2_4_3X_Can_prostate_cancer_be_found_e arly_36.asp?sitearea=.)

14. Abramson EL, Krumholz HM, Vaccarino V. Moderate Alcohol Consumption and Risk of Heart Failure Among Older Persons. The Journal of American Medical Association 2001;285(15):19717.WS

15. Scales CD Jr. Curtis LH, Norris RD, Schulman KA, Dahm P, Moul JW. Relationship between body mass index and prostate cancer screening in the United States. J Urol 2007;177(2):493-8. [PubMed: 17222617]

16. Poortinga W. The prevalence and clustering of four major lifestyle risk factors in an English adult population. Preventive Medicine 2007;44(2):124-8. [PubMed: 17157369]

17. Gattellari M, Young JM, Ward JE. GP and patient predictors of PSA screening in Australian general practice. Fam Pract 2003;20(3):294-303. [PubMed: 12738699]

18. Close DR, Li S, Patterson RE. Associations of Demographic and Health-related Characteristics with Prostate Cancer Screening in Washington State. Cancer Epidemiology, Biomarkers \& Prevention 1998;7:627-30.KA, aWE

19. Chan EC, Vernon SW, Ahn C, Greisinger A. Do men know that they have had a prostate-specific antigen test? Accuracy of self-reports of testing at 2 sites. Am J Public Health 2004;94(8):1336-8. [PubMed: 15284039]

20. Lamplugh M, Gilmore P, Quinlan T, Cornford P. PSA testing: are patients aware of what lies ahead? Ann R Coll Surg Engl 2006;88(3):284-8. [PubMed: 16720000] 


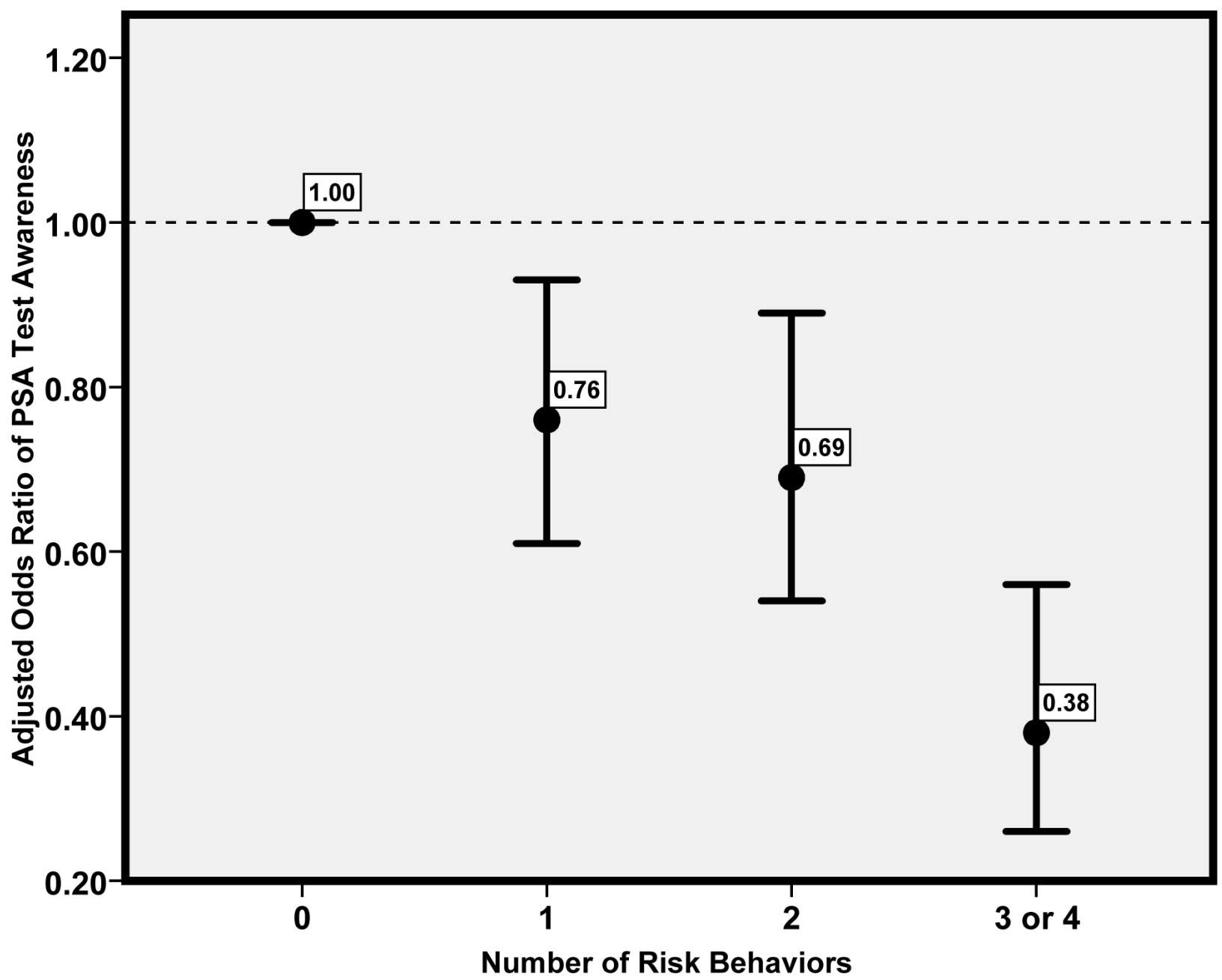

Figure 1. Effect of Clustering of Health Risk Behaviors on Odds Ratios of PSA Awareness OR adjusted for age, race, percentage of life spent in the US, English language speaking ability, income, education, marital status, health insurance, usual source of care other than ER, number of physician visits in the past year, self reported health status, and colorectal cancer screening. 
Table 1

Characteristics of the Study Population and Prevalence of PSA Awareness: CHIS 2003

\begin{tabular}{|c|c|c|c|}
\hline Characteristics & $\begin{array}{c}\text { Population } \\
\mathrm{n}=7,297(\%)^{a}\end{array}$ & $\begin{array}{c}\text { Prevalence of } \\
\text { PSA } \\
\text { Awareness (\%) }\end{array}$ & p-value ${ }^{b}$ \\
\hline \multicolumn{4}{|l|}{ Age } \\
\hline $50-54$ & 26.5 & 65.9 & \multirow[t]{5}{*}{0.0001} \\
\hline $55-59$ & 20.6 & 75.3 & \\
\hline $60-64$ & 15.8 & 77.0 & \\
\hline $65-69$ & 11.0 & 77.1 & \\
\hline $70+$ & 26.0 & 74.3 & \\
\hline \multicolumn{4}{|l|}{ Race/Ethnicity } \\
\hline White & 66.0 & 80.7 & \multirow[t]{7}{*}{$<0.0001$} \\
\hline Latino & 13.6 & 58.9 & \\
\hline Pacific Islander & 0.3 & 85.8 & \\
\hline American Indian /Alaska Native & 1.0 & 76.8 & \\
\hline Asian & 10.5 & 48.0 & \\
\hline African American & 6.0 & 68.6 & \\
\hline Other, Single/Multiple Race & 2.6 & 62.1 & \\
\hline \multicolumn{4}{|l|}{ Income } \\
\hline $0-99 \%$ FPL & 9.1 & 47.2 & \multirow[t]{4}{*}{$<0.0001$} \\
\hline 100-199\% FPL & 14.7 & 55.8 & \\
\hline $200-299 \%$ FPL & 14.0 & 64.0 & \\
\hline$\geq 300 \% \mathrm{FPL}$ & 62.3 & 82.9 & \\
\hline \multicolumn{4}{|l|}{ Education } \\
\hline$<$ High school & 17.9 & 51.6 & \multirow[t]{4}{*}{$<0.0001$} \\
\hline High school & 17.8 & 69.8 & \\
\hline Some college & 23.8 & 75.1 & \\
\hline$\geq$ College degree & 40.4 & 82.8 & \\
\hline \multicolumn{4}{|l|}{ Marital Status } \\
\hline Married & 73.1 & 74.9 & \multirow[t]{3}{*}{0.02} \\
\hline Divorced/separated/ widow/partner & 21.2 & 67.5 & \\
\hline Never married & 5.7 & 69.6 & \\
\hline \multicolumn{4}{|l|}{ Insurance Status } \\
\hline Currently Uninsured & 7.8 & 54.0 & \multirow[t]{3}{*}{0.12} \\
\hline Uninsured Any Period Past Year & 2.0 & 51.2 & \\
\hline Insured Past 12 Months & 90.1 & 75.2 & \\
\hline \multicolumn{4}{|l|}{ Physician Visits in Past Year } \\
\hline 0 & 12.6 & 58.8 & \multirow[t]{4}{*}{0.0001} \\
\hline $1-2$ & 32.2 & 73.2 & \\
\hline $3-4$ & 23.5 & 74.9 & \\
\hline 5 or more & 31.8 & 77.1 & \\
\hline \multicolumn{4}{|l|}{ Self-Reported Health Status } \\
\hline Excellent & 18.3 & 79.6 & 0.03 \\
\hline
\end{tabular}




\begin{tabular}{|c|c|c|c|}
\hline Characteristics & $\begin{array}{c}\text { Population } \\
\mathrm{n}=7,297(\%)^{a}\end{array}$ & $\begin{array}{c}\text { Prevalence of } \\
\text { PSA } \\
\text { Awareness }(\%)^{a}\end{array}$ & p-value ${ }^{b}$ \\
\hline Very Good & 28.2 & 80.5 & \\
\hline Good & 27.1 & 73.1 & \\
\hline Fair & 18.0 & 60.2 & \\
\hline Poor & 8.5 & 61.0 & \\
\hline \multicolumn{4}{|l|}{ Colonic Cancer Screening } \\
\hline Within Past 5 Years & 52.9 & 81.1 & $<0.0001$ \\
\hline Not Within Past 5 Years & 16.6 & 78.2 & \\
\hline Never & 30.5 & 56.3 & \\
\hline
\end{tabular}




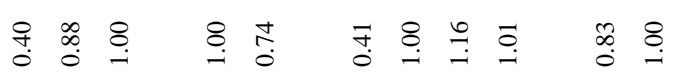

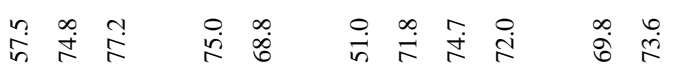

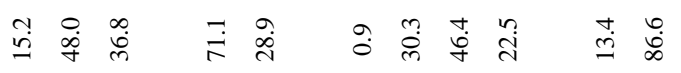

